\title{
Impacto do tratamento quimioterápico na qualidade de vida de pacientes oncológicos
}

Impact of chemotherapy treatment on the quality of life of patients with cancer Impacto del tratamiento de quimioterapia en la calidad de vida de pacientes oncológicos

Fernanda Modesto Silveira ${ }^{1}$ iD https://orcid.org/0000-0003-1764-4649 Anneliese Domingues Wysocki ${ }^{1}$ io https://orcid.org/0000-0002-8381-9999

Roberto Della Rosa Mendez ${ }^{1}$ io https://orcid.org/0000-0003-2934-6526

Silvana Barbosa Pena ${ }^{1}$ iD https://orcid.org/0000-0001-8276-014X

Edirlei Machado dos Santos ${ }^{1}$ io https://orcid.org/0000-0002-1221-0377

Silmara Malaguti-Toffano ${ }^{2}$ io https://orcid.org/0000-0002-9080-9123

Vinícius Batista dos Santos ${ }^{3}$ io https://orcid.org/0000-0001-5130-5523

Mariana Alvina dos Santos ${ }^{1}$ id hitps://orcid.org/0000-0002-7940-6673

Silveira FM, Wysocki AD, Mendez como citar: Santos EM, Malaguti-Toffano S, et al. Impacto do tratamento quimioterápico na qualidade de vida de pacientes oncológicos. Acta Paul Enferm. 2021;34:eAPE00583.

DOI

http://dx.doi.org/10.37689/actaape/2021A000583

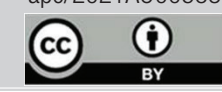

Descritores

Qualidade de vida; Neoplasias; Quimioterapia adjuvante

Keywords

Quality of life; Neoplasms; Chemotherapy, adjuvante

Descriptores

Calidad de vida; Neoplasias; Quimioterapia adyuvante

\section{Submetido \\ 30 de Março de 2020 \\ Aceito \\ 20 de Agosto de 2020}

\section{Autor correspondente}

Mariana Alvina dos Santos Email: marifamema@yahoo.com.br

\section{Resumo}

Objetivo: Avaliar a qualidade de vida relacionada à saúde (QVRS) de pacientes oncológicos antes e três meses após o início do tratamento quimioterápico.

Métodos: Tratou-se de um estudo de coorte prospectivo, exploratório. Foi utilizado um instrumento para caracterização sociodemográfica e clínica dos pacientes e outro para avaliação da QVRS denominado EORTC QLQ-C30, sendo este constituído por três escalas: funcionalidade, sintomas e saúde global. Participaram 79 indivíduos atendidos em um ambulatório para realização de quimioterapia. Testes estatísticos foram realizados para comparar o efeito do tratamento quimioterápico em relação à qualidade de vida.

Resultados: 0 instrumento EORTC QLQ-C30 indicou adequada confiabilidade nos dois momentos de avaliação. No que tange à escala de funcionalidade, as funções física e cognitiva apresentaram melhora; e a função emocional, piora após três meses do tratamento. A escala de sintomas revelou piora, após três meses do início da quimioterapia, no que diz respeito aos sintomas de fadiga, náusea, dispneia, perda de apetite e diarreia.

Conclusão: Neste estudo, os domínios mais afetados, após três meses de tratamento quimioterápico, estavam relacionados às funções da escala funcional e dos sintomas, portanto, intervenções multiprofissionais devem ser implementadas a esta população visando ao controle de tais variáveis.

\section{Abstract}

Objective: To assess the health-related quality of life (HRQLL) of patients with cancer before and three months after starting chemotherapy treatment.

Methods: This is a prospective and exploratory cohort study. An instrument was used for sociodemographic and clinical characterization of patients and another for assessing HRQOL called EORTC QLQ-C30. This instrument consists of functional, symptom, and global health status scales. Seventy-nine individuals attended at an outpatient clinic for chemotherapy. Statistical tests were performed to compare side effects of chemotherapy treatment on quality of life.

Results: The EORTC QLQ-C30 indicated adequate reliability in two assessment moments. Concerning the functional scale, physical functioning and cognitive functioning improved, and emotional functioning worsened after three months of treatment. The symptom scale worsened three months after starting chemotherapy with respect to the symptoms of fatigue, nausea, dyspnea, appetite loss, and diarrhea.

Conclusion: The most affected domains, after three months of chemotherapy treatment, were related to functional and symptom scales' functioning; therefore, multidisciplinary interventions should be implemented for this population in order to control such variables. 


\section{Resumen}

Objetivo: Evaluar la calidad de vida relacionada con la salud (CVRS) de pacientes oncológicos antes del inicio del tratamiento de quimioterapia y tres meses después. Métodos: Se trató de un estudio de cohorte prospectivo, exploratorio. Fue utilizado un instrumento para la caracterización sociodemográfica y clínica de los pacientes y otro para la evaluación de la CVRS denominado EORTC QLQ-C30, que está compuesto por tres escalas: funcionalidad, síntomas y salud global. Participaron 70 individuos atendidos en consultorios externos para la realización de quimioterapia. Se realizaron pruebas estadísticas para comparar el efecto del tratamiento de quimioterapia con relación a la calidad de vida.

Resultados: El instrumento EORTC QLQ-C30 indicó fiabilidad adecuada en los dos momentos de evaluación. En lo que atañe a la escala de funcionalidad, la función física y la cognitiva presentaron mejora, y la función emocional empeoró después de tres meses de tratamiento. La escala de síntomas reveló empeoramiento, luego de tres meses del inicio de la quimioterapia, en lo que se refiere a los síntomas de fatiga, náuseas, disnea, pérdida de apetito y diarrea.

Conclusión: En este estudio, los dominios más afectados luego de tres meses de tratamiento de quimioterapia se relacionaron con funciones de la escala funcional y de los síntomas; por lo tanto, intervenciones multiprofesionales deben ser implementadas en esta población con el objetivo de controlar dichas variables.

\section{Introdução}

O câncer é um conjunto de doenças caracterizadas pelo crescimento indiscriminado de células podendo se disseminar pela corrente sanguínea ou pelo sistema linfático, dessa forma, acometendo outros tecidos e órgãos. Essas células são agressivas e dividem-se com rapidez formando uma massa celular, nomeada tumor, ${ }^{(1,2)}$ sendo uma das principais causas de mortalidade por doenças no mundo. ${ }^{(3)}$

Esse aumento deve-se, em parte, ao envelhecimento e crescimento populacional, como também à mudança na distribuição e na prevalência dos fatores de risco para o câncer, sobretudo, aqueles relacionados ao desenvolvimento socioeconômico. Por conseguinte, observa-se uma mudança dos principais tipos de cânceres identificados nos países em desenvolvimento, com a queda dos tipos de câncer relacionados a infecçôes e aumento daqueles associados à melhor condição socioeconômica e ao conjunto de hábitos de vida, tais como a falta de atividades física, inadequada alimentação, entre outros. (4) A mais recente estimativa mundial aponta que ocorreram 18 milhóes de casos novos de câncer e 9,6 milhóes de óbitos no ano de 2018. ${ }^{(4)}$

No Brasil, a estimativa para o triênio de 20202022 aponta que ocorrerão 625 mil casos novos de câncer (450 mil, excluindo os casos de câncer de pele não melanoma). O câncer de pele não melanoma será o mais incidente $(177 \mathrm{mil})$, seguido pelos cânceres de mama e próstata (66 mil cada), cólon e reto (41 mil), pulmão (30 mil) e estômago (21 mil). (4) Os tipos de câncer mais frequentes em homens, à exceção do câncer de pele não melanoma, serão próstata $(29,2 \%)$, cólon e reto $(9,1 \%)$, pulmáo (7,9\%), estômago (5,9\%) e cavidade oral (5,0\%); e, nas mulheres, os cânceres de mama (29,7\%), cólon e reto $(9,2 \%)$, colo do útero $(7,4 \%)$, pulmão $(5,6 \%)$ e tireoide $(5,4 \%)$ estarão entre os mais frequentes. ${ }^{(4)}$

Essas estatísticas mostram que o país continuará com números elevados de cânceres de mama feminina, próstata, cólon e reto e pulmão, embora também apresente elevadas taxas para cânceres de estômago, chamando atenção a incidência do câncer de pele não melanoma.

A escolha do método de tratamento depende da natureza e extensão da doença. A cirurgia (ressecção), a radioterapia e a quimioterapia são as estratégias mais frequentes para o manejo da doença oncológica. ${ }^{(2)}$ A quimioterapia elimina as células cancerígenas e também afeta as células normais, sendo considerada uma terapêutica com elevados efeitos colaterais. ${ }^{(5)}$

O tratamento interfere nas condiçóes físicas do paciente, ocasionando o agravamento de sintomas físicos como insônia, náusea, fadiga, perda de apetite, alopecia, além de interferir na capacidade para realização das atividades de vida diária (independência e autonomia), nos relacionamentos interpessoais e na forma como o paciente analisa esta situação e a si mesmo, acrescido ao risco de desequilíbrio emocional e psicológico, pois há o receio de viver com as dificuldades que a doença e o tratamento provocam e pelo próprio estigma de o diagnóstico do câncer estar associado à morte. Todas essas possíveis alteraçôes podem afetar as expectativas para o futuro e, consequentemente, a qualidade de vida. ${ }^{(6)}$

A Qualidade de Vida (QV) é definida como "a percepção do indivíduo de sua posição na vida, no âmbito do sistema cultural e de valores em que vive e em relação a suas metas, expectativas, padróes e preocupaçôes", ${ }^{(7)}$ ou seja, a QV está relacionada à satisfa- 
ção na vida familiar, amorosa, social e ambiental. $\mathrm{O}$ termo qualidade de vida relacionada à saúde (QVRS) é mais utilizado na área da saúde, pois está ligado a mudanças na percepção, no estado funcional, emocional e aos fatores sociais influenciados pela saúde, por doenças e tratamentos, ou seja, está relacionado à intervenção em saúde e às enfermidades. ${ }^{(8)}$

Avaliar o efeito do tratamento oncológico na QVRS dos pacientes com diagnóstico de câncer pode auxiliar a equipe multiprofissional, especialmente os profissionais de enfermagem, no planejamento de intervençóes que minimizem esses possíveis efeitos, portanto, diante do exposto este estudo teve por objetivo avaliar a qualidade de vida relacionada à saúde (QVRS) de pacientes oncológicos antes e três meses após o início do tratamento quimioterápico.

\section{Métodos}

Trata-se de um estudo de coorte prospectivo, exploratório, com abordagem quantitativa, realizado no serviço de oncologia de um hospital beneficente na cidade de Três Lagoas-MS. Foram convidados a participar da pesquisa todos os pacientes que iniciaram o tratamento quimioterápico na referida instituição de junho de 2018 a junho de 2019. A amostra foi de conveniência, composta por 79 participantes que atenderam aos seguintes critérios de inclusão: indicação de tratamento quimioterápico e ser maior de 18 anos. Foram excluídos aqueles com inabilidade de comunicação.

O local da pesquisa funciona diariamente das 7 às 17 horas, onde são realizados procedimentos como quimioterapia, imunoterapia, consulta médica, administração de medicação, curativos e sondagens. $\mathrm{O}$ atendimento, neste local, destina-se a pacientes do Sistema Único de Saúde (SUS), com convênios de saúde e particular e trata-se de um serviço de extrema importância na macrorregiáo, tendo em vista a agilidade com a qual se dão o início dos tratamentos e das intervençóes e visto que outros centros de referência de oncologia são afastados.

Foram recrutados todos os pacientes que iniciaram tratamento quimioterápico no período da pesquisa. Depois de selecionados, os indivíduos elegíveis foram convidados a participar da pesquisa; e aqueles que aceitaram foram inclusos no estudo e assinaram o Termo de Consentimento Livre e Esclarecido (TCLE). Dois pesquisadores foram treinados para a realização das entrevistas de coleta dos dados, que foram efetivadas na sala de quimioterapia (na poltrona onde o paciente receberia o tratamento), no primeiro dia - antes de iniciar a infusão da quimioterapia (T1) - e três meses após (T2), em ambas as ocasiōes, as entrevistas se deram de forma individual e com uso de biombo para assegurar a privacidade dos envolvidos.

Para a coleta dos dados foram utilizados dois instrumentos: o questionário de caracterização sociodemográfico e clínico; e o European Organization for Research and Treatment of Cancer Quality of Life Questionnaire - Core 30 (EORTC QLQ - C30, version 3), específico para pacientes com câncer, validado para o português. No primeiro momento foram aplicados ambos os questionários antes de iniciar a quimioterapia (T1) e, no segundo momento (T2), três meses após o T1, foi aplicado apenas o EORTC, além de recolhidas informações sociodemográficas às quais houvesse ocorrido mudanças no período de três meses. ${ }^{(9-13)}$

O EORTC QLQ C30 conta com 30 questôes divididas em três escalas principais: escala funcional, que possui cinco subescalas que avaliam as funçôes física, cognitiva, emocional, social e de desempenho de papel; a escala de sintomas, que avalia questóes referentes à dor, à fadiga, à náusea/ao vômito, à dispneia, à insônia, à perda de apetite, à constipação, à diarreia e a preocupaçóes financeiras; e a escala de qualidade de vida/saúde global. Para o cálculo do escore de cada escala do EORTC QLQ 30, procedeu-se à transformação linear do escore (0 a 100), de acordo com as recomendaçóes do EORTC, sendo que, para as escalas funcional e saúde global/QV, quanto maior o escore, melhor o funcionamento; e, na escala de sintomas, dando-se o oposto, sendo o escore mais próximo ao zero a ausência de sintomas. ${ }^{(9)}$

Os dados foram inicialmente digitados em planilha do Microsoft Excel, e a análise estatística foi realizada pelo programa SPSS versão 22.0. Para a 
análise estatística descritiva foi realizado o cálculo de frequência relativa e absoluta para as variáveis categóricas e o cálculo de média e desvio padrão para as variáveis quantitativas.

No que tange à análise da confiabilidade do EORTC QLQ C30 foi calculada a consistência interna de cada escala do instrumento e do instrumento global pelo alfa de Cronbach, sendo considerada adequada consistência interna quando obtido um alfa de Cronbach > 0,70. Para a análise do impacto do tratamento quimioterápico na $\mathrm{QV}$ foi aplicado o teste $t$-Student para amostras pareadas comparando-se as médias de cada escala do instrumento e de seus indicadores. Foi considerado um valor $p<0,05$ como estatisticamente significativo.

O estudoatendeuà Resolução n. ${ }^{\circ}$ 466/12 do CNS e foi aprovado pelo Comitê de Ética da Universidade Federal de Mato Grosso do Sul com o Parecer n. ${ }^{\circ}$ 2.621.175 e CAAE 87369118.5.0000.0021.

\section{Resultados}

A amostra foi constituída de 79 participantes, sendo a maioria do gênero feminino, com idade acima de 60 anos, com companheiro, com renda de até 4 SM, com escolaridade até o Ensino Fundamental completo ou incompleto, católica e da raça branca, conforme tabela 1 . No que diz respeito aos hábitos de vida, a maior parte da amostra era de fumantes e ex-fumantes, não consumia bebida alcoólica, não praticava atividade física, não trabalhava nem exercia atividade remunerada no período da pesquisa, náo recebia ajuda para realização das atividades de vida diária e não fazia uso de medicação contínua.

Os tipos de câncer mais prevalentes foram intestino $(28 \%)$, mama e ginecológico (28\%), cabeça e pescoço (15\%), sistema gastrointestinal (11\%), pulmão $(8 \%)$ e outros $(9 \%)$. Os tumores descritos na categoria "outros" foram linfoma, bexiga, rim e desconhecido. A maioria não tinha o diagnóstico de metástase (77\%), o tempo de diagnóstico foi entre zero e quatro meses $(71 \%)$ e grande parte não fazia acompanhamentos nutricional $(72 \%)$ e psicológico (86\%), conforme a tabela 2.
Tabela 1. Características sociodemográficas dos participantes em tratamento quimioterápico

\begin{tabular}{|c|c|}
\hline Características & $\mathrm{n}(\%)$ \\
\hline \multicolumn{2}{|l|}{ Gênero } \\
\hline Feminino & $48(61)$ \\
\hline Masculino & $31(39)$ \\
\hline \multicolumn{2}{|l|}{ Idade } \\
\hline de 20 a 39 anos & $9(11)$ \\
\hline de 40 a 49 anos & $11(14)$ \\
\hline de 50 a 59 anos & $19(24)$ \\
\hline$\geq 60$ anos & $40(51)$ \\
\hline \multicolumn{2}{|l|}{ Renda (SM) } \\
\hline 0 a 2 & $39(49)$ \\
\hline 3 a 4 & $32(41)$ \\
\hline$>5$ & $8(10)$ \\
\hline \multicolumn{2}{|l|}{ Estado conjugal } \\
\hline Com companheiro(a) & $48(61)$ \\
\hline Sem companheiro(a) & $31(39)$ \\
\hline \multicolumn{2}{|l|}{ Escolaridade } \\
\hline Analfabeto & $3(4)$ \\
\hline Ensino Fundamental completo/incompleto & $45(57)$ \\
\hline Ensino Médio completo/incompleto & $27(34)$ \\
\hline Ensino Superior completo/incompleto & $4(5)$ \\
\hline \multicolumn{2}{|l|}{ Religião } \\
\hline Católico(a) & $55(70)$ \\
\hline Evangélico(a) & $20(25)$ \\
\hline Outras & $4(5)$ \\
\hline \multicolumn{2}{|l|}{ Raça } \\
\hline Branca & $38(49)$ \\
\hline Parda & $31(39)$ \\
\hline Amarela & $1(1)$ \\
\hline Negra & $9(11)$ \\
\hline \multicolumn{2}{|l|}{ Tabagismo } \\
\hline Fumante/ex-fumante & $47(60)$ \\
\hline Nunca fumou & $32(40)$ \\
\hline \multicolumn{2}{|l|}{ Etilismo } \\
\hline Consome bebida alcoólica & $6(8)$ \\
\hline Não consome bebida alcoólica & 73(92) \\
\hline \multicolumn{2}{|l|}{ Atividade física } \\
\hline Pratica atividade física & $33(42)$ \\
\hline Não pratica atividade física & $46(58)$ \\
\hline \multicolumn{2}{|l|}{ Trabalha atualmente } \\
\hline Sim & 24(30) \\
\hline Não & $55(70)$ \\
\hline \multicolumn{2}{|l|}{ Ajuda para fazer atividades diárias } \\
\hline $\operatorname{Sim}$ & $8(10)$ \\
\hline Não & $71(90)$ \\
\hline \multicolumn{2}{|l|}{ Medicações } \\
\hline Não toma medicamento & $31(40)$ \\
\hline Até dois medicamentos & $22(27)$ \\
\hline Mais de três medicamentos & $17(21)$ \\
\hline Não lembra & $9(12)$ \\
\hline
\end{tabular}

SM - salário mínimo; referência: R\$ 954,00 e 998,00, anos vigentes 2018 e 2019

$\mathrm{Na}$ avaliação da consistência interna do instrumento EORTC QLQ-30, obteve-se um alfa de Cronbach de 0,86. No que diz respeito a cada escala do instrumento, obteve-se como alfa de Cronbach, no primeiro e segundo momentos, na escala fun- 
Tabela 2. Características clínicas dos pacientes em tratamento quimioterápico

\begin{tabular}{lc}
\hline Características & $\mathrm{n}(\%)$ \\
\hline Tipo de neoplasia & \\
Intestino & $22(28)$ \\
Mama e ginecológico & $22(28)$ \\
Cabeça e pescoço & $12(15)$ \\
Pulmão & $6(8)$ \\
Sistema gastrointestinal & $9(11)$ \\
Outros & $7(9)$ \\
Metástase & \\
Sim & $18(23)$ \\
Não & $61(77)$ \\
Tempo de diagnóstico (meses) & \\
0 a 4 & $56(71)$ \\
5 a 11 & $17(21)$ \\
Mais de 12 & $6(8)$ \\
Acompanhamento nutricional & \\
Sim & $22(28)$ \\
Não & $57(72)$ \\
Acompanhamento psicológico & \\
Sim & $11(14)$ \\
Não & $68(86)$ \\
\hline
\end{tabular}

cional, de 0,87 e 0,88; na escala de sintomas, 0,83 e 0,79 ; e, na escala saúde global/QV, 0,87 e 0,88 . Ao comparar o escore da escala funcional final e da escala de saúde global/Qualidade de Vida antes e três meses após o início da quimioterapia, detectou-se que náo houve diferença estatisticamente significativa, porém, quanto à escala de sintomas, verificou-se piora significativa desse indicador após três meses da quimioterapia $(\mathrm{p}<0,001)$, conforme a tabela 3 .

Tabela 3. Escore médio do instrumento QLQ-C30 obtido no pré-tratamento (T1) e três meses após a quimioterapia (T2)

\begin{tabular}{lccc}
\hline Escore médio das dimensões & $\begin{array}{c}\text { T1 } \\
\text { das escalas }\end{array}$ & $\begin{array}{c}\text { T2 } \\
\text { Média (DP) }\end{array}$ & p-value \\
\hline Escala funcional final & $35,44(23,0)$ & $35,67(24,18)$ & 0,87 \\
\hline Função física & $32,74(28,4)$ & $36,11(28,59)$ & $<0,01$ \\
\hline Função cognitiva & $17,51(26,8)$ & $20,89(28,67)$ & 0,04 \\
\hline Função emocional & $49,47(33,8)$ & $42,72(33,87)$ & $<0,01$ \\
\hline Função social & $26,16(29,5)$ & $32,28(30,11)$ & 0,06 \\
Desempenho de papel & $41,35(39,9)$ & $38,61(37,41)$ & 0,47 \\
\hline Escala de sintomas final & $26,68(21,3)$ & $34,53(20,49)$ & $<0,001$ \\
Dor & $39,66(40,5)$ & $33,33(38,3)$ & 0,09 \\
Fadiga & $34,74(34,6)$ & $41,35(29,9)$ & 0,01 \\
Náusea & $9,92(24,3)$ & $34,60(33,4)$ & $<0,01$ \\
Dispneia & $9,70(23,3)$ & $17,30(31,5)$ & 0,04 \\
Insônia & $41,35(43,7)$ & $40,93(41,3)$ & 0,94 \\
Perda de apetite & $29,11(38,9)$ & $44,73(39,5)$ & $<0,01$ \\
Constipação & $29,54(43,6)$ & $30,80(39,5)$ & 0,74 \\
Diarreia & $6,75(20,2)$ & $13,50(26,9)$ & 0,05 \\
Preocupações financeiras & $27,00(35,0)$ & $41,77(39,39)$ & $<0,01$ \\
Saúde global/QV final & $68,14(27,63)$ & $64,66(23,87)$ & 0,19 \\
\hline
\end{tabular}

DP - desvio padrão; QV - qualidade de vida; * teste $t$-Student para amostras pareadas
$\mathrm{Na}$ análise dos itens de cada dimensão da escala funcional foi evidenciado melhora após três meses do tratamento na funçáo física $($ p.<0,01) e cognitiva (p. 0,04) da escala funcional, porém com piora no tocante à função emocional (p.<0,01). $\mathrm{Na}$ escala de sintomas final, averiguaram-se pioras nos sintomas de fadiga (p.0,01), náusea $(\mathrm{p}<0,01)$, dispneia (p. 0,04), perda de apetite (p.<0,01), diarreia (p.0,05) e preocupaçóes financeiras $(\mathrm{p} .<0,01)$, conforme tabela 3 .

\section{Discussão}

O perfil sociodemográfico da amostra revelou características semelhantes às de outros estudos, ${ }^{(14,15)}$ na qual houve predomínio do gênero feminino, com idade média de 57 anos e pessoas que conviviam com companheiro, dados que convergem conforme as informaçôes do Instituto Nacional do Câncer e apontaram maior incidência do câncer em pessoas com mais de 40 anos de idade. ${ }^{(4)}$

Conquanto os participantes do estudo não representem a população brasileira por ter sido uma amostra de um hospital de médio porte, as características clínicas identificadas neste estudo tiveram semelhança com as que foram encontradas em outra investigação com idosos no Brasil com diagnóstico médico de câncer, no qual a maioria era mulher e com renda de até dois salários mínimos. ${ }^{(9)}$ No presente estudo, a maior quantidade de pacientes não estava trabalhando no momento da coleta de dados devido à aposentadoria ou afastados em decorrência do diagnóstico médico e do tratamento, reforçando o conceito de que o tratamento do câncer pode levar ao afastamento precoce das atividades laborais, contribuindo para $\mathrm{o}$ alto percentual de licença médica. ${ }^{\left({ }^{8)}\right.}$

Quanto à escolaridade e renda, predominaram baixo nível escolar e socioeconômico, similar a um estudo desenvolvido em Recife - PE, ${ }^{(16)}$ o que pode justificar os dados identificados de piora pertinentes a preocupaçôes financeiras após três meses do início do tratamento quimioterápico, pois a piora na escala de sintomas pode comprometer a capacidade para a realização de atividades laborais remuneradas. ${ }^{(15,16)}$ 
A maioria dos participantes era fumante e ex-fumante $(60 \%)$, sobrepondo-se aos pacientes que não fumavam. Segundo o INCA, o tabagismo é um fator de risco para diversos cânceres, além de doenças cardiovasculares e respiratórias. ${ }^{(17)}$

No tocante às características do estilo de vida, a maioria (58\%) náo praticava atividade física, achado que foi similar ao de uma pesquisa em que as mulheres não praticavam atividade física em mais de $56,5 \%$ dos casos. ${ }^{(15)}$ Esse fato é algo importante a ser observado, visto que a International Agency for Research on Cancer (IARC) considera o sedentarismo um fator de risco para o desenvolvimento de câncer de mama. ${ }^{(15)} \mathrm{O}$ hábito de realizar uma atividade física, além de ser um fator de proteção, promove o bem-estar físico e emocional para os pacientes durante o tratamento. ${ }^{(16)}$

A maior incidência foi câncer de intestino e mama/ginecológico, e, ao comparar com um estudo em Pernambuco, a maioria, nesse, foi de mama $(32,3 \%)$, pulmão $(22,6 \%)$ e próstata $(16,1 \%) .{ }^{(8)}$ Dados da estimativa do INCA também corroboram o estudo em tela, visto que o perfil dos cânceres mais encontrados no Brasil, no biênio 2018-2019, foram de próstata, pulmão, mama feminina e cólon e reto. ${ }^{(4)}$ À exceção do câncer de pele não melanoma, os tipos de câncer mais incidentes em homens foram: próstata $(31,7 \%)$, pulmão $(8,7 \%)$, intestino $(8,1 \%)$, estômago $(6,3 \%)$ e cavidade oral $(5,2 \%)$; e, nas mulheres, os cânceres de mama $(29,5 \%)$, intestino $(9,4 \%)$, colo do útero $(8,1 \%)$, pulmão $(6,2 \%)$ e tireoide $(4,0 \%) .{ }^{(4)} \mathrm{O}$ câncer de próstata não foi apontado nos resultados do estudo em tela, pois, no ambulatório no qual se desenvolveu a pesquisa, quase todos os pacientes realizavam apenas o tratamento cirúrgico e de imunoterapia; e os poucos casos mais avançados faziam quimioterapia. A alta prevalência do câncer de mama e intestino justificou-se devido ao maior número de mulheres presentes neste estudo.

$\mathrm{Na}$ escala funcional, não houve mudanças significativas comparando os dois momentos de coleta de dados, indicando que o domínio de funcionalidade não foi afetado pelo tratamento quimioterápico, mas permaneceu com a média baixa, indicando prejuízos antes mesmo do início do tratamento. ${ }^{(17)}$
Um estudo com idosos relata resultados em que sucedeu piora no domínio de desempenho físico, o que pode ser explicado pela quantidade de idosos e, portanto, ele já era previamente alterado em razão de outras comorbidades e condiçáo física, o que está de acordo com este estudo, no qual se deu maior prevalência de idosos. ${ }^{(8)}$

Os achados do presente estudo demonstraram melhora das funçôes física e cognitiva três meses após o início do tratamento quimioterápico. Esses dados divergiram dos resultados identificados em outro estudo, ${ }^{(18)}$ no qual se expóe que houve piora na função física em decorrência dos afeitos adversos do tratamento quimioterápico, então, alterando, por conseguinte, a qualidade de vida dos pacientes.

Uma justificativa, no que concerne ao presente estudo, a inferir quanto à melhora física relatada pelos pacientes seria que o ocorrido tenha-se relacionado com a melhora da função cognitiva, visto que o paciente que apresente melhora da função cognitiva e compreensão do seu problema pode sentir-se mais confiante para realizar suas atividades diárias, com isso, tendo uma percepção de melhora física. Outro ponto que poderia justificar a melhora relatada seria que $49 \%$ dos pacientes deste estudo tinham idade inferior a 60 anos, o que seria um fator protetor para a disposição física, apesar do diagnóstico. ${ }^{(14)}$

No tocante à melhora da função cognitiva relatada pelos pacientes, poder-se-ia justificá-la porque, ao iniciar a quimioterapia, estavam em maior contato com os profissionais de saúde responsáveis pelo seu tratamento e, desse modo, recebiam mais informações e esclarecimentos pertinentes às suas dúvidas relativamente à doença e ao tratamento. Cabe ressaltar a importância da equipe de enfermagem que, devido à sua proximidade ao paciente durante o tratamento, pode estabelecer vínculos de confiança e facilitar-lhes esclarecimentos. ${ }^{(15,16)}$

A função emocional foi avaliada por meio de perguntas que abrangiam ansiedade, preocupaçáo, irritação e depressão, sendo um dos domínios que assinalaram pioras na segunda avaliação. Esse achado também é evidenciado em uma pesquisa realizada em Recife, com mulheres com câncer de mama, em que esse domínio relata mais alteração. ${ }^{(16)}$ Por ter vários fatores envolvidos, a relação entre câncer de mama e 
depressão é complexa e, muitas vezes, não é identificada e diagnosticada, sendo que a alteração emocional pode permanecer durante todo o tratamento e até mesmo após seu término. ${ }^{(18,19)}$

Neste estudo, percebeu-se que a maioria dos participantes não fazia acompanhamento psicológico antes e após o diagnóstico do câncer. $\mathrm{O}$ risco de alteraçóes emocionais graves pode ter gerado um elevado prejuízo na QVRS desses pacientes e na continuidade do tratamento. $\mathrm{O}$ enfermeiro é quem está à frente e age como interlocutor da equipe de saúde, podendo, então, liderar diferentes formas de intervenção diante de situações que possam vir a causar prejuízo na vida do paciente, além de planejar açóes que resultem maior segurança e qualidade da assistência. ${ }^{(20)}$

Em um estudo ${ }^{(21)}$ são discriminadas uma série de alteraçôes pertinente ao campo emocional em pacientes com diagnóstico de câncer, tais como vontade de melhorar o autoconceito, desespero, risco de comprometimento da vontade humana, distúrbio pessoal de identidade, sensação de impotência, risco de solidão, baixa autoestima situacional, distúrbio de imagem corporal, processos familiares interrompidos, vontade de melhorar o processo familiar, negação ineficiente, estresse, tristeza, isolamento social, portanto, é importante que o enfermeiro esteja atento e identifique essas possíveis alteraçóes para que possa implementar ações de enfermagem de forma independente ou em conjunto com outros profissionais de saúde, tais como auxílio na melhora da autopercepção, promoção de esperança e melhora do enfrentamento, fortalecimento da autoestima, melhoria da socialização, aumento da segurança, apoio emocional e escuta ativa, além de incluir os familiares para que auxiliem e apoiem no processo do tratamento, influenciando de maneira positiva a lidar com o estresse e as dificuldades. ${ }^{(21)}$

Fadiga, dispneia, náusea, perda de apetite, diarreia e preocupaçóes financeiras foram os sintomas que apresentaram piora após três meses do tratamento quimioterápico. Segundo um estudo realizado com pacientes com câncer de mama, essas alteraçóes revelam-se a partir da segunda sessão de quimioterapia e também quando o diagnóstico é mais tardio, o que pode estar relacionado a um tratamento mais agressivo e a uma dificuldade de adquirir medicaçóes que controlem esses sintomas. ${ }^{(14)}$ A prevalência de fadiga foi elevada neste estudo, resultado que se assemelha aos observados em investigaçóes realizadas em outros países. ${ }^{(22,23)}$

Insônia, fadiga, dispneia e perda de apetite e dor são os sintomas mais prevalentes em estudo realizado, em 2016, com 162 pacientes com câncer, ${ }^{(24)}$ assim, podendo-se inferir que a severidade dos sintomas pode afetar tanto funçóes físicas quanto emocionais, conforme evidenciado no presente estudo.

A relação entre fadiga e câncer é comum e, apesar de possuir múltiplos fatores, é relatada como um estado intenso de esgotamento, com duração e intensidade maior que a fadiga comum. Ela pode afetar os domínios funcionais, reduzir a satisfação pessoal e a qualidade de vida, ${ }^{(25)}$ interferir nas atividades do dia a dia do paciente e no próprio tratamento, pois, quando muito intensa, pode haver a necessidade de interromper o tratamento ou reduzir a dose do quimioterápico. ${ }^{(11)}$

A perda de apetite pode dar-se tanto por causa dos aspectos físicos relacionados ao surgimento de sintomas - alteração no paladar, náusea, mucosites e enterites - como no que tange aos aspectos emocionais - alteraçôes de humor em pacientes com câncer associadas diretamente com o aparecimento de outros sintomas, tais como perda de massa ponderal, constipação, diarreia, fadiga e piora do padrão respiratório, além da piora na qualidade de vida e mortalidade. Por conseguinte, intervençôes multiprofissionais devem ser realizadas de forma imediata a esses pacientes, iniciando-se com a análise da ingesta alimentar para identificação de situaçôes de risco nutricional e incorporação precoce de suplementos alimentares. ${ }^{(26,27)}$

No estudo em tela, houve redução nos níveis de insônia e dor após três meses do tratamento, embora sem significância estatística. Uma hipótese para a melhora desses sintomas pode ter sido a ação do quimioterápico de forma positiva no prognóstico da doença ou também pela ação de medicaçóes utilizadas durante o tratamento para controle dos sintomas. Ambos são sintomas comuns em pacientes com câncer, e a dor pode interferir na qualidade do sono, conforme um estudo que apresenta dados divergen- 
tes aos detectados neste, pois relata que insônia e dor aumentam durante o tratamento quimioterápico e impactam na qualidade de vida. ${ }^{(10)}$ Outros estudos confirmam a associação entre a presença de dor no câncer e prejuízos à qualidade de vida. ${ }^{(28,29)}$

Foi observado que o escore avaliado de Saúde Global/QV final diminuiu após três meses do tratamento em comparação entre T1 e T2, porém sem significância estatística. A Saúde Global/QV foi avaliada por meio de uma autoavaliação do paciente, sendo que este dava a nota conforme sua percepção a respeito da sua qualidade de saúde no geral. A maioria dos participantes, conquanto se tratasse de uma situação de enfermidade, não considerou a Saúde Global/QV ruim. Não existe uma forma exata de quantificá-la, pois ela é subjetiva e pessoal, portanto, o que é uma vida com boa qualidade para uma pessoa pode não ser para outra. A QVRS é caracterizada pouco satisfatória em um estudo, ${ }^{(5)}$ porém, em outro, não se aponta alteração entre o antes e depois, exceto para pacientes com câncer de próstata. ${ }^{(8)} \mathrm{A}$ QVRS é, de igual modo, considerada boa pelos participantes de outra investigação, cujos resultados também apontam alteraçóes em outros domínios. ${ }^{(15,16,30)}$

Este estudo viabilizou contribuiçóes importantes para a área da enfermagem porque consultas de acompanhamento de enfermagem rotineiras a esta população seriam benéficas na identificação precoce de alteraçóes de funcionalidade e de sintomas, além de instrumentalizar o profissional com dados para uso e implementação das classificaçôes de enfermagem. Conhecer a QVRS, nesse grupo de pacientes, permite que os enfermeiros planejem a execução do cuidado nos serviços de quimioterapia de modo a individualizar e melhorar a qualidade da assistência.

Este estudo apresentou limitaçóes: a amostra foi de conveniência e os dados foram coletados em único cenário, o que impossibilitou a generalização dos resultados.

\section{Conclusão}

Os dados identificados neste estudo revelaram que os domínios mais afetados, após três meses de tratamento quimioterápico, relacionavam-se à função emocional da escala funcional e aos sintomas de fadiga, náusea, perda de apetite, dispneia, diarreia e preocupaçóes financeiras da escala de sintomas, bem como foi identificado melhora nas funçóes física e cognitiva da escala funcional. Posto isso, foi possível inferir que conhecer a ação dos efeitos do tratamento quimioterápico na QVRS propicia auxílio à equipe de enfermagem no planejamento da assistência e reabilitação; desse modo, visando-se identificar e realizar intervençôes para diminuir os prejuízos do tratamento, assim como viabilizar a elaboração de estratégias para assegurar que esses pacientes tenham uma vida de qualidade ao longo desse período.

\section{Agradecimentos}

A Pesquisa foi financiada pelo Programa Institucional de Bolsas de Iniciação Cientifica - PIBIC (2018/2019) - Conselho Nacional de Desenvolvimento Científico e Tecnológico (CNPq). O presente trabalho foi realizado com apoio da Universidade Federal de Mato Grosso do Sul - UFMS/MEC - Brasil.

\section{Colaborações}

Silveira FM, Wysocki AD, Mendez RDR, Pena SB, Santos EM, Malaguti-Toffano S, Santos VB e Santos MA declaram com a concepção do estudo, coleta e interpretação dos dados, revisão crítica relevante do conteúdo intelectual e aprovação da versão final a ser publicada.

\section{Referências}

1. Instituto Nacional de Câncer (INCA). 0 que é o câncer? [Internet]. Rio de Janeiro: INCA; 2019. [citado 2019 Ago 8]. Disponível em: https:// www.inca.gov.br/0-que-e-cancer

2. Floriano DF, Ribeiro PF, Maragno AC, Rossi K, Simões PW. Oral complications in patients treated with radiotherapy or chemotherapy in a hospital in Santa Catarina. Rev Odontol Univ Cid São Paulo. 2017;29(3):230-6.

3. Panis C, Kawasaki AC, Pascotto CR, Justina EY, Vicentini GE, Lucio LC, Prates RT. Critical review of cancer mortality using hospital records and potential years of life lost. einstein (São Paulo). 2018;16(1):eA04018. 
4. Instituto Nacional de Câncer (INCA). Estimativa 2020: incidência de câncer no Brasil [Internet]. Rio de Janeiro: INCA; 2020 [citado 2020 Abr 24]. Disponível em: http://www.inca.gov.br/estimativa/2020/ estimativa-2020.pdf

5. Gomes RA, Coelho AC, Moura DC, Cruz JSC, Santos KB. Evaluation of the quality of life of patients with onco-haematological disease in chemotherapy. Rev enferm UFPE. 2018 [;12(5):1200-5.

6. Santos AL, Franco HH, Vasconcelos FC. [Association between nutritional status and psychological changes in patients with gastrointestinal cancer]. Braspen J. 2017;32(4):362-8. Portuguese.

7. Correia RA, Bonfim CV, Ferreira DK, Furtado BM, Costa HV, Feitosa KM, et al. Quality of life after treatment for cervical cancer. Esc Anna Nery. 2018;22(4):e20180130.

8. Ferreira ML, Souza Al, Ferreira LO, Moura JF, Junior Jl. Qualidade de vida relacionada à saúde de idosos em tratamento quimioterápico. Rev Bras Geriatr Gerontol. 2015;18(1):165-77.

9. Paiva CE, Carneseca EC, Barroso EM, de Camargos MG, Alfano AC, Rugno FC, et al. Further evaluation of the EORTC QLQ-C30 psychometric properties in a large Brazilian cancer patient cohort as a function of their educational status. Support Care Cancer. 2014;22(8):2151-60.

10. Amorim JR, Silva IA, Shimizu IS. Avaliação da qualidade de sono em pacientes com câncer de mama em quimioterapia. Rev Bras Mastologia. 2017;27(1):3-7.

11. Avelar JM, Nicolussi AC, Toneti BF, Sonobe HM, Sawada NO. Fatigue in patients with head and neck cancer undergoing radiation therapy: a prospective study. Rev Lat Am Enfermagem. 2019;27:e3168.

12. Aaronson NK, Ahmedzai S, Bergman B, Bullinger M, Cull A, Duez NJ, et al. The European Organization for Research and Treatment of Cancer QLQ-C30: a quality-of-life instrument for use in international clinical trials in oncology. J Natl Cancer Inst. 1993;85(5):365-76.

13. Pais-Ribeiro J, Pinto C, Santos C. Validation study of the portuguese version of the QLC-C30-V.3. Psicol Saude Doencas. 2008;9(1):89 102

14. Garcia SN, Félix JV, Montovani MF, Maftum MA, Kalinke LP. Quality of life of women with breast cancer receiving chemotherapeutic treatment. Rev Baiana Enferm. 2017;31(2):e17489.

15. Andrade V, Sawada NO, Barichello E. Quality of life in hematologic oncology patients undergoing chemotherapy. Rev Esc Enferm USP. 2013 Apr;47(2):355-61.

16. Coelho RC, Panobianco MS, Guimarães PR, Guimarães PR, Maftum MA, Santos PN, Kalinke LP. Adjuvant and neo adjuvant chemotherapy and the implications in the quality of life women with breast cancer. Rev Enferm UFPE Online. 2017;11(Supl 11):4732-40.

17. Instituto Nacional de Câncer (INCA). Prevenção e fatores de risco [Internet]. Rio de Janeiro: INCA; 2018. [citado 2019 Ago 8]. Disponível em: https://www.inca.gov.br/causas-e-prevencao/prevencao-efatores-de-risco

18. Villar RR, Fernández SP, Garea CC, Pillado MT, Barreiro VB, Martín CG. Quality of life and anxiety in women with breast cancer before and after treatment. Rev Lat Am Enfermagem. 2017;25(0):e2958.

19. Mansano-Schlosser TC, Ceolim MF. Longitudinal variations of sleep quality in women with breast cancer. Acta Paul Enferm. 2016;29(5):595-602.

20. Spotten LE, Corish CA, Lorton CM, Ui Dhuibhir PM, O'Donoghue NC, O'Connor B, et al. Subjective and objective taste and smell changes in cancer. Ann Oncol. 2017;28(5):969-84.

21. Ribeiro JP, Cardoso LS, Pereira CM, Silva BT, Bubolz BK, Castro CK. Nursing care in oncology hospitalized patients: diagnosis and interventions related to psychosocial and psychospiritual needs. Rev Fund Care Online. 2016;8(4):5136-42.

22. Jean-Pierre P, Morrow GR, Roscoe JA, Heckler C, Mohile S, Janelsins $\mathrm{M}$, et al. A phase 3 randomized, placebo-controlled, double-blind, clinical trial of the effect of modafinil on cancer-related fatigue among 631 patients receiving chemotherapy: a University of Rochester Cancer Center Community Clinical Oncology Program Research base study. Cancer. 2010 Jul;116(14):3513-20.

23. Hofman M, Ryan JL, Figueroa-Moseley CD, Jean-Pierre P, Morrow GR. Cancer-related fatigue: the scale of the problem. Oncologist. 2007;12(S1 Suppl 1):4-10.

24. Salvetti MG, Machado CS, Donato SC, Silva AM. Prevalence of symptoms and quality of life of cancer patients. Rev Bras Enferm. 2020;73(2):e20180287.

25. Borges JA, Quintão MM, Chermont SS, Mendonça Filho HT, Mesquita ET. Fatigue: A Complex Symptom and its Impact on Cancer and Heart Failure. Int J Cardiovasc Sci. 2018;31(4):433-42.

26. Mattox TW. Cancer Cachexia: cause, diagnosis, and treatment. Nutr Clin Pract. 2017 0ct;32(5):599-606.

27. Arends J, Bachmann P, Baracos V, Barthelemy N, Bertz H, Bozzetti $F$, et al. ESPEN guidelines on nutrition in cancer patients. Clin Nutr. 2017;36(1):11-48

28. Cardoso G, Graca J, Klut C, Trancas B, Papoila A. Depression and anxiety symptoms following cancer diagnosis: a cross-sectional study. Psychol Health Med. 2016;21(5):562-70.

29. Barrett M, Chu A, Chen J, Lam KY, Portenoy R, Dhingra L. Quality of life in community-dwelling chinese american patients with cancer pain. $\mathrm{J}$ Immigr Minor Health. 2017;19(6):1442-8.

30. Avelino CU, Cardoso RM, Aguiar SS, Silva MJ. Assessment of quality of life in patients with advanced non-small cell lung carcinoma treated with a combination of carboplatin and paclitaxel. J Bras Pneumol. 2015;41(2):133-42. 